\title{
EXTRA-ARTICULAR FRACTURE OF THE MEDIAL END OF THE CLAVICLE ASSOCIATED WITH TYPE IV ACROMIOCLAVICULAR DISLOCATION: CASE REPORT
}

Mário Chaves Correa', Lucas Braga Jacques Gonçalves², Jose Carlos Souza Vilela², Igor Lima Leonel ${ }^{3}$, Lincoln Paiva Costa ${ }^{4}$, Ronaldo Percopi de Andrade ${ }^{5}$

\section{ABSTRACT}

Fractures of the clavicle and acromioclavicular dislocations are very common injuries when they occur separately. The combination of an acromioclavicular dislocation and a fracture of the lateral third of the clavicle is not rare. However, there are very few reported cases of acromioclavicular dislocations associated with fractures of the middle third of the clavicle; those associated with fractures of the medial third are even rarer. We report the case of an adult male who suffered an acromioclavicular dislocation (type IV) associated with a displaced extra-articular fracture of the medial end of the clavicle (Almann group 3) in a cycling accident. The patient was treated during the acute phase with open reduction and internal fixation of the two lesions. At the clinical evaluation 12 months after the surgery, the patient was asymptomatic, with full active and passive mobility, and normal strength and endurance of the shoulder girdle. Radiographs and a three-dimensional CT scan showed persistent posterosuperior subluxation of the acromioclavicular joint and anatomical consolidation of the clavicular fracture.

Keywords - Acromioclavicular Joint; Dislocation/surgery; Shoulder Fractures

\section{CASE REPORT}

A right-handed 41-year-old man suffered a fall from a bicycle, with trauma to the posterolateral face of his right shoulder. On examination in the emergency department, the injury site was seen to be abraded, with diffuse edema in the ipsilateral clavicular region. It was intensely painful, with crepitation on palpation of the clavicle and acromioclavicular region (Figure 1). Neurovascular examination of the extremity of the arm gave normal results. Radiographic examination revealed a displaced extra-articular fracture of the medial extremity of the clavicle, and marked widening of the acromioclavicular joint (Figure 2). Three-dimensional computed tomography showed a comminuted extra-articular fracture of the medial extremity of the right clavicle, with anteroinferior displacement of the lateral fragment, and type IV acromioclavicular dislocation (Figures 3a, 3b and 3c).

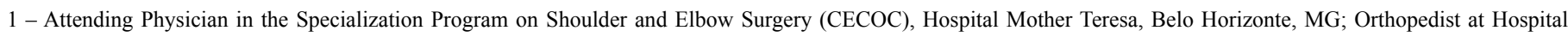
Governador Israel Pinheiro (HGIP-IPSEMG), Belo Horizonte, MG, Brazil.

2 - Attending Physician in the Specialization Program on Shoulder and Elbow Surgery (CECOC), Hospital Mother Teresa, Belo Horizonte, MG, Brazil.

3 - Specialization Program on Shoulder and Elbow Surgery (CECOC), Hospital Mother Teresa, Belo Horizonte, MG, Brazil.

4 - Orthopedist at Hospital Mother Teresa, Belo Horizonte, MG, Brazil.

5 - Head of the Orthopedics and Traumatology Service, Hospital Mother Teresa, Belo Horizonte, MG, Brazil.

Work performed at Hospital Madre Teresa, Belo Horizonte, MG, Brazil.

Correspondence: Rua Chile 245/404, 30310-670 Belo Horizonte, MG, Brazil. E-mail: mrccf@uol.com.br

Work received for publication: July 24, 2010; accepted for publication: October 29, 2010.
}

The authors declare that there was no conflict of interest in conducting this work 


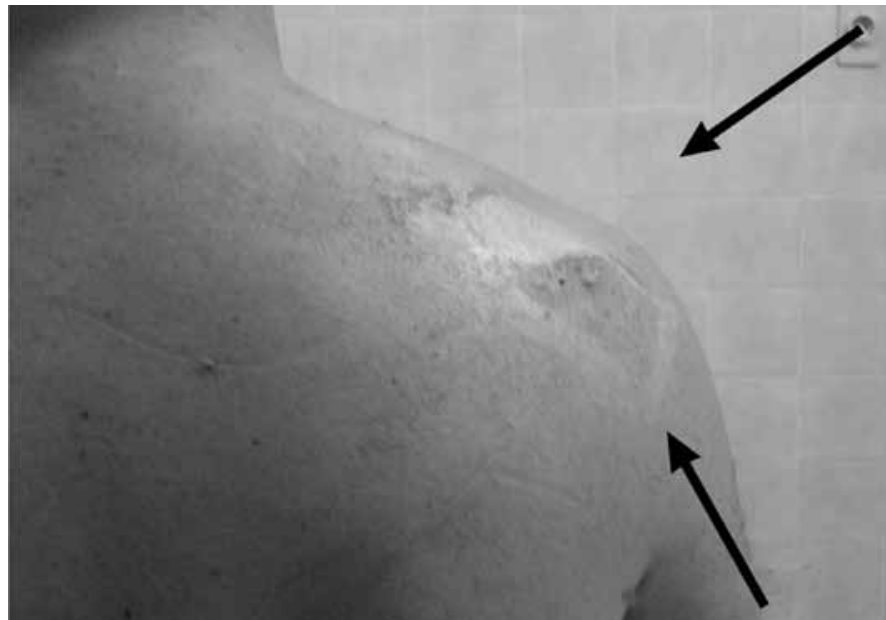

Figure 1 - Abrasion of the posterolateral region of the shoulder and edema in the acromioclavicular region, thus showing the trauma mechanism.

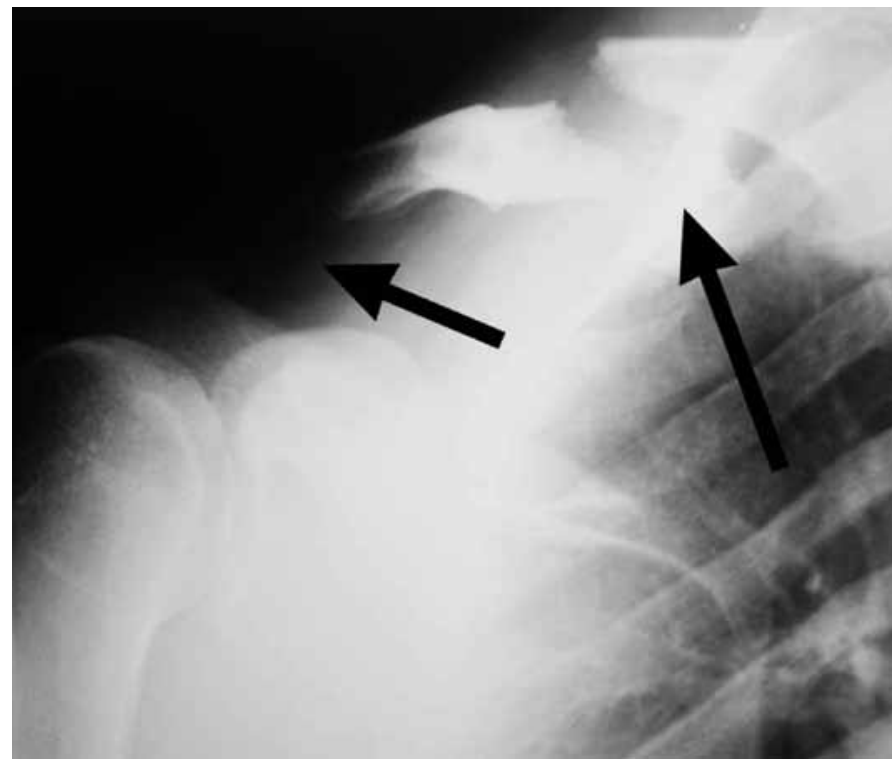

Figure 2 - Radiograph showing displaced extra-articular medial fracture of the clavicle and marked widening of the acromioclavicular joint.

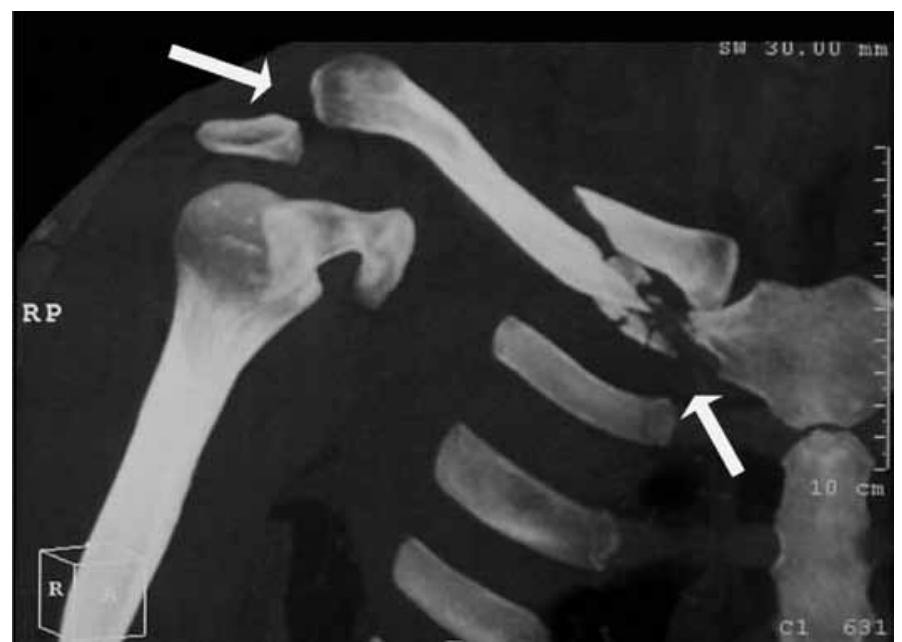

Figure 3a - Three-dimensional computed tomography on the shoulder, clearly demonstrating the two lesions. Anterior view.

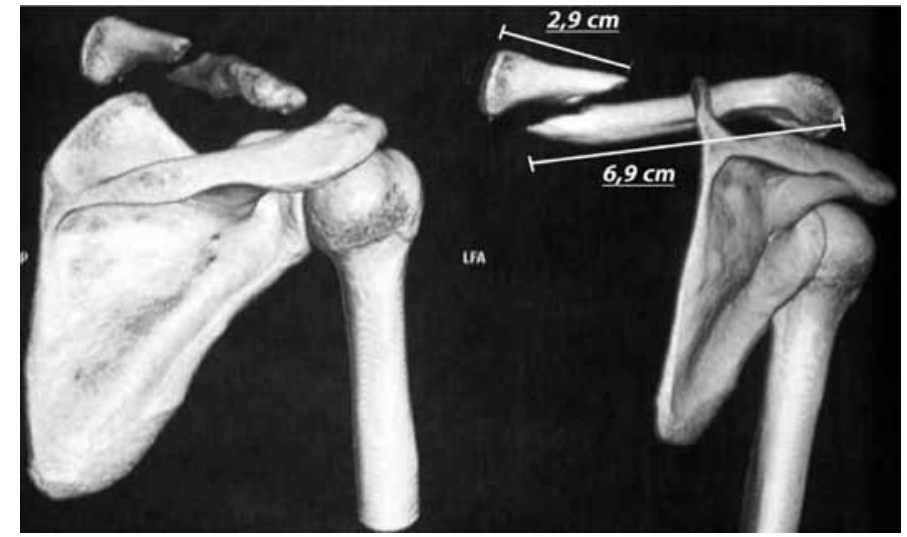

Figure $\mathbf{3 b}$ - Posterior view.

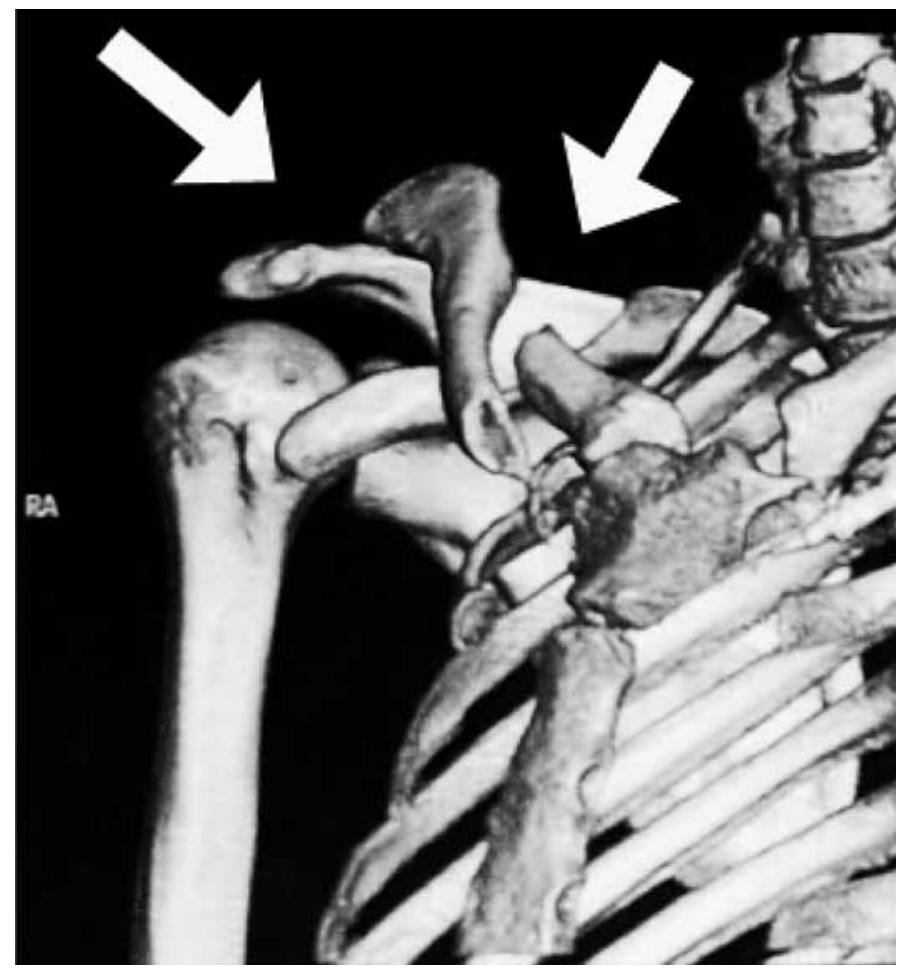

Figure $3 c-$ View from above.

It was decided to administer surgical treatment, which was done seven days after the trauma. The patient was put into a semi-seated position ("deckchair position"). A horizontal incision following the line of the clavicle confirmed the tomographic findings and showed that the trapezius-deltoid fascia was torn. The fracture was reduced anatomically and fixed using a molded reconstruction plate, which was placed on the anterosuperior surface of the clavicle, with three 3.5 $\mathrm{mm}$ cortical screws in the medial fragment and five 3.5 $\mathrm{mm}$ cortical screws in the lateral fragment. It was possible to reduce the acromioclavicular joint as an open surgical procedure, but this could not be maintained. The coracoclavicular ligaments were found to be totally 
torn and shredded, and it was impossible to suture them. The acromioclavicular joint was then reduced as an open procedure and stabilized using a coracoclavicular tie by means of two $5 \mathrm{~mm}$ anchors (loaded with double no. 2 braided polyester thread) that were fixed at the coracoid process. The first suture was passed around the entire circumference of the clavicle and tied off at the top. The second suture was passed through two bone tunnels connecting the upper and lower cortical bone of the clavicle and was also tied off on the top surface (Figure 4). The trapezius-deltoid fascia was reconstructed using no. 2 braided polyester thread. The reduction and stabilization of the acromioclavicular joint were considered to be appropriate and were confirmed by means of radioscopy (Figure 5). The arm was immobilized in a sling for five weeks, and then physiotherapy was started. In a radiographic control performed in the second month after the operation, posterosuperior subluxation of the clavicle was observed (Figure 6). However, this did not have any functional or cosmetic repercussion, and the patient had not noticed it. It was decided to continue with the rehabilitation, which extended for four months. After six months, the patient had recovered full movement of the shoulder and did not have any spontaneous pain or pain on palpation of the acromioclavicular joint. He had resumed cycling practice at the same level as before the trauma and had started muscle training. After 12 months, he continued to be free from symptoms and was regularly practicing physical exercises. There was a small and almost imperceptible prominence in the acromioclavicular joint (Figures 7a, 7b, 7c and 7d). A new computed tomography scan confirmed that the posterosuperior subluxation of the acromioclavicular joint had persisted, but without any degenerative changes to the joint (Figures 8a, 8b and 8c).

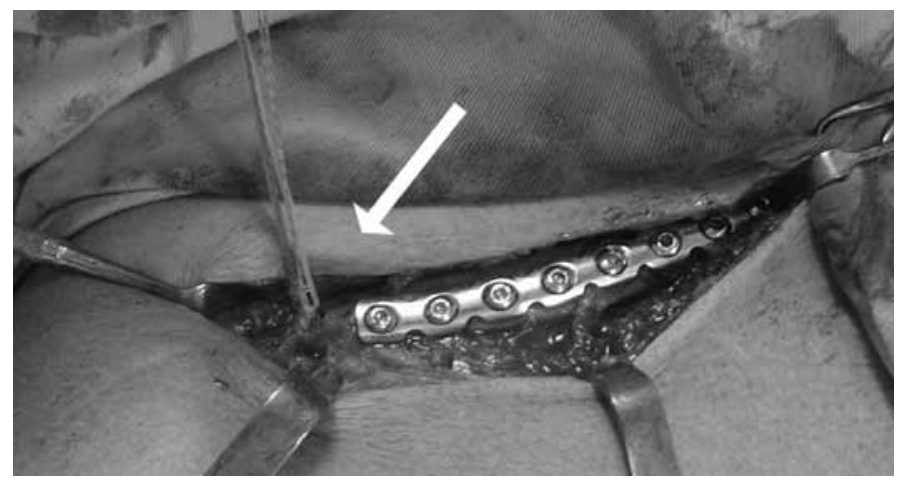

Figure 4 - Perioperative appearance: premolded plate and coracoclavicular tie with two anchors that were doubly loaded with no. 2 braided polyester thread and were fixed to the coracoid process).

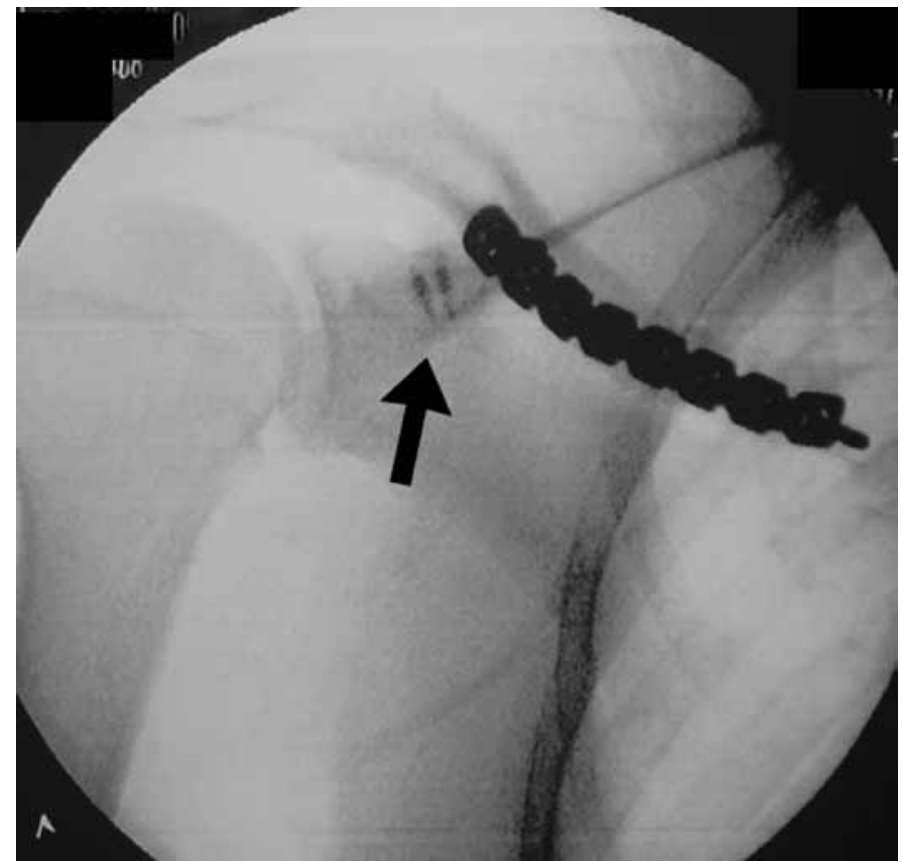

Figure 5 - Perioperative radioscopy. Note the anatomical reduction of the clavicular fracture and the normal coracoclavicular distance.

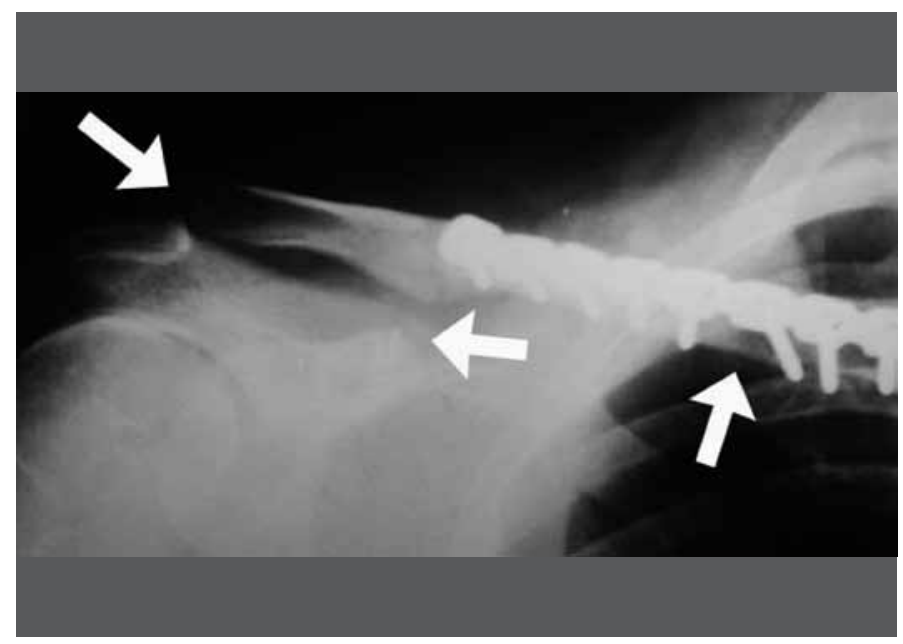

Figure 6 - Radiograph produced in the second month after the operation. Subluxation of the acromioclavicular joint and anatomical consolidation of the clavicular fracture.

\section{DISCUSSION}

Separately, fractures of the clavicle and acromioclavicular dislocation are among the most frequent traumatic lesions of the scapular belt ${ }^{(1-3)}$. The combination of acromioclavicular dislocation and a fracture in the lateral third of the clavicle is not rare ${ }^{(2,4)}$. However, very few cases of acromioclavicular dislocation associated with fractures of the middle third of the clavicle have been described ${ }^{(4,5)}$; those associated with fractures of the medial third are even $\operatorname{rarer}^{(3,6)}$. 

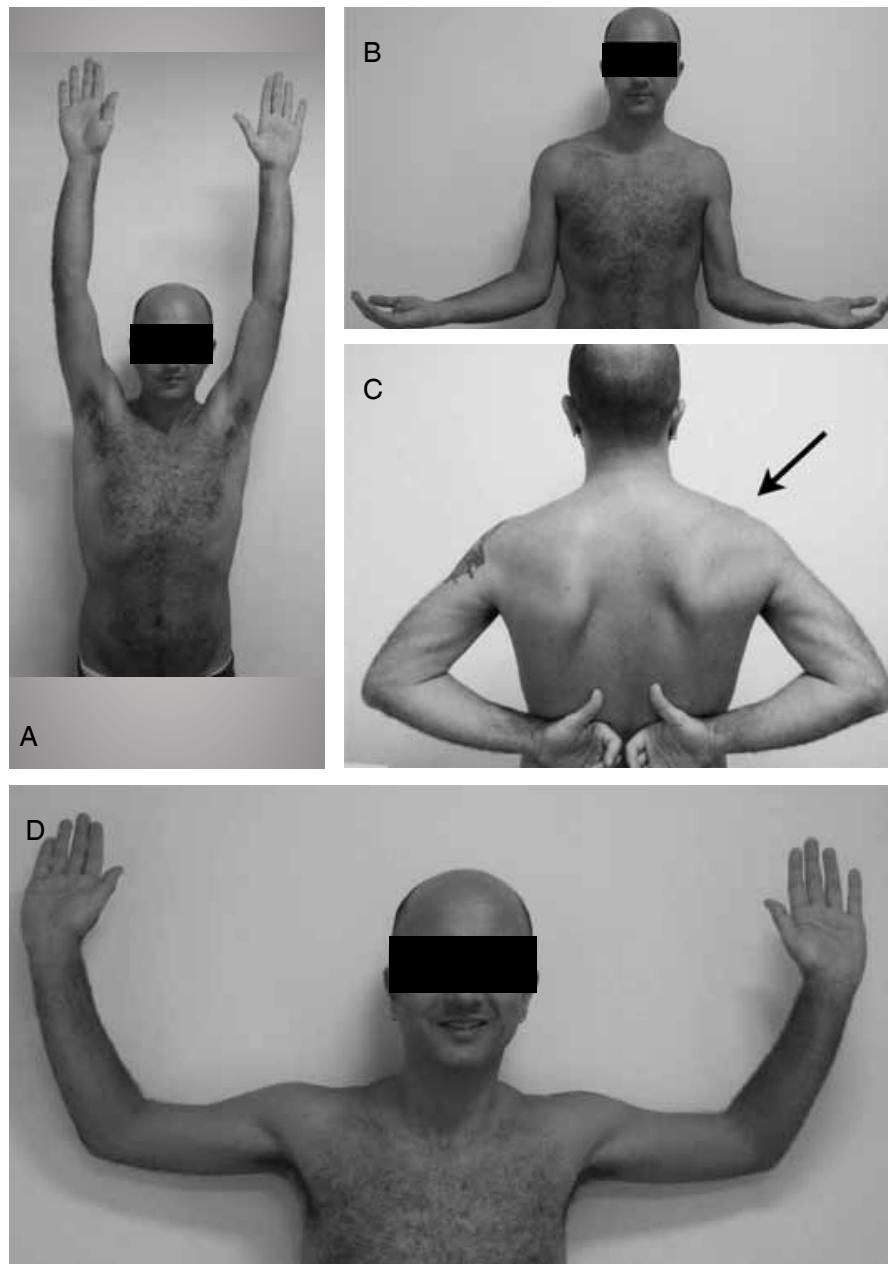

Figure 7 - Functional and cosmetic result, one year after the operation. (A) Anterior elevation. (B) External rotation in adduction. (C) Internal rotation in adduction; note the slight prominence of the lateral extremity of the right clavicle. (D) External rotation in abduction.

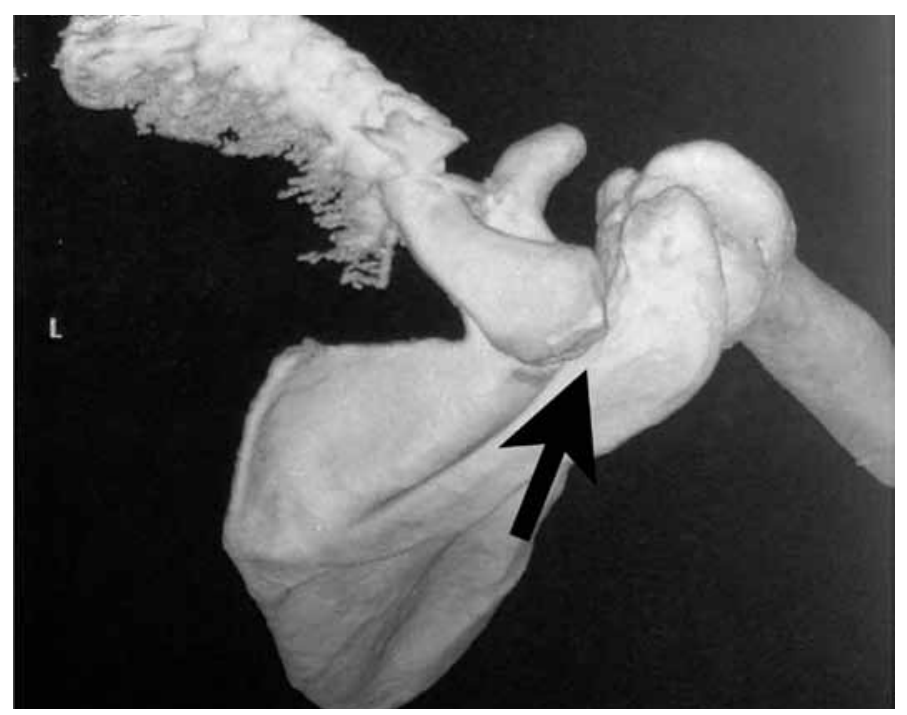

Figure $\mathbf{8 A}$ - Three-dimensional computed tomography on the scapular belt after the operation, looking down on the posterosuperior subluxation of the clavicle. The irregularities observed in the clavicle and coracoid process are image artifacts caused by the presence of metallic material (plate and screws, and anchors, respectively).

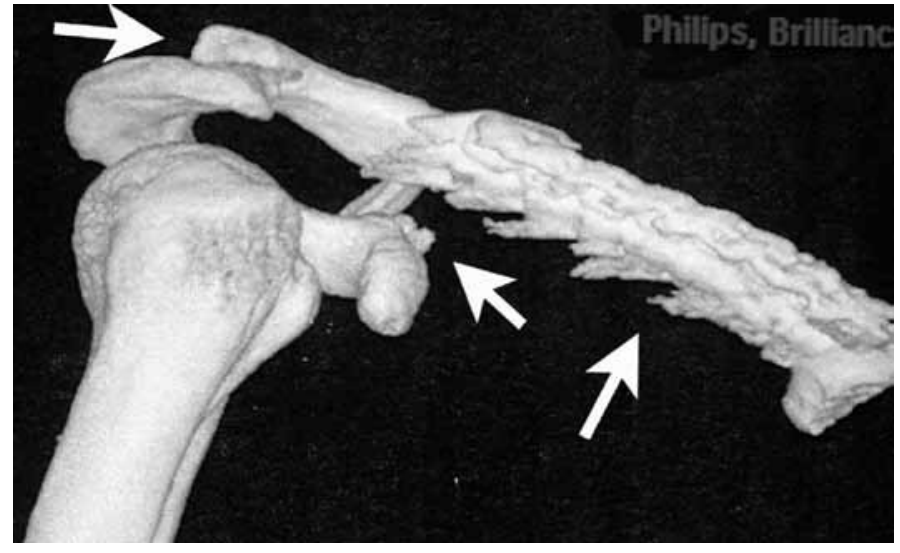

Figure 8B - Three-dimensional computed tomography on the scapular belt after the operation, with anterior view.

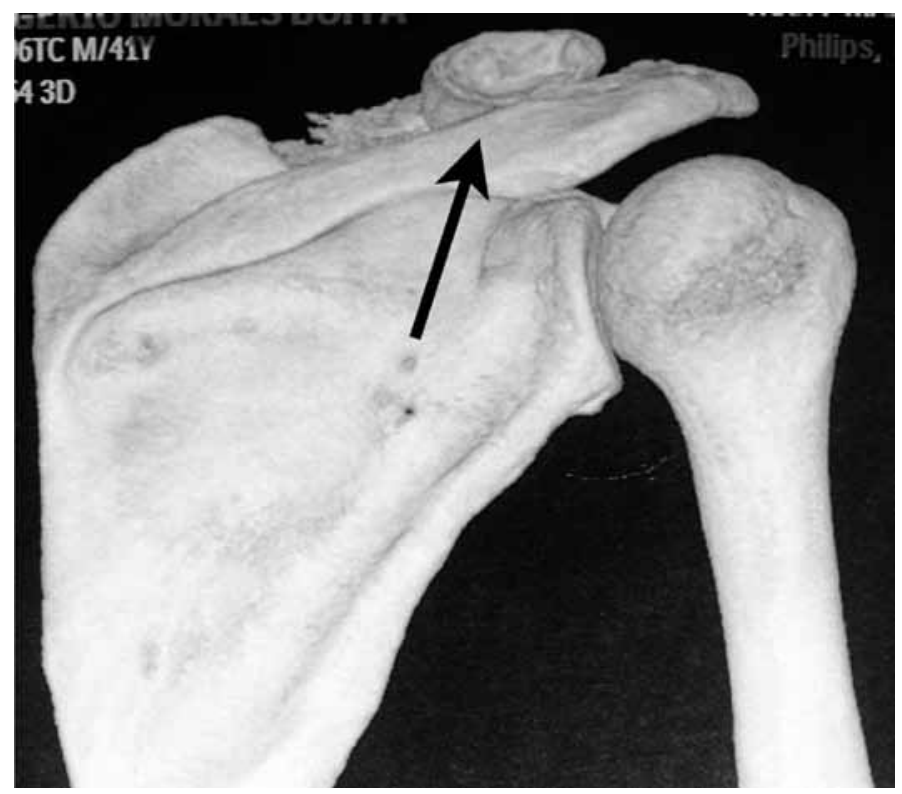

Figure $\mathbf{8 C}$ - Three-dimensional computed tomography on the scapular belt after the operation, with posterior view.

Almann classified fractures of the clavicle into three groups: type I, in the middle third, with incidence of $80 \%$; type II, in the lateral third, with incidence of $15 \%$; and type III, in the medial third, with incidence of $5 \%{ }^{(1)}$.

Rockwood et al ${ }^{(1)}$ classified acromioclavicular dislocations into six types based on their degree and the direction of the displacement of the scapular belt in relation to the lateral extremity of the clavicle ${ }^{(7)}$.

Historically, fractures of the clavicle have mostly been treated non-surgically ${ }^{(6)}$. However, more recently, studies have shown unsatisfactory functional results after non-surgical treatment of certain highly displaced and/or shortened fractures. There is an increasing trend towards favoring surgical treatment in such cases, espe- 
cially among young and/or physically active patients ${ }^{(4)}$. These fractures can be stabilized by means of open reduction and internal fixation using an intramedullary wire or plates and screws. This last technique seems to be the preferred option for most authors.

The treatment for acromioclavicular dislocations is well established in the literature. Types I and II lesions are generally treated non-surgically and generally evolve without functional or esthetic sequelae. Dislocations of types IV, V and VI are considered to be formal indications for surgery ${ }^{(1,2,7)}$. Controversy still exists in relation to the treatment for type III lesions, but studies published more recently have favored non-surgical treatment of these lesions in the acute phase ${ }^{(1,8)}$. Although there is a consensus regarding surgical indications for acromioclavicular dislocations of types IV, V and VI, the same cannot be said in relation to the best way to stabilize them $^{(1,5,9)}$. It has been estimated that between 50 and 70 different techniques exist, including direct repairs with or without reinforcement using synthetic material or autologous or heterologous tendon grafts, coracoclavicular ties with or without anchors, transarticular wires, ligament transpositions with resection of the lateral extremity of the clavicle, tendon transpositions, coracoclavicular screws, hook plates, or combinations of one or several of these procedures ${ }^{(1,10)}$. Numerous complications have been described in relation to all of them, and one of the commonest complications is partial or total loss of the reduction, which in most cases evolves without clinical repercussions, independent of the technique used ${ }^{(1,9)}$.

Associations between acromioclavicular dislocation and fractures in the lateral third of the ipsilateral clavicle are not uncommon. On the other hand, acromioclavicular dislocations associated with fractures of the middle third $^{(5)}$ and, especially, with fractures of the medial third, are extremely rare ${ }^{(6)}$. We did not find any specific classification in the literature, or any well-established recommendations for treating acromioclavicular dislocations associated with ipsilateral fractures of the middle or medial third of the clavicle in adult patients ${ }^{(3)}$, possibly because of the rarity of such associations.

Lancourt et al apud Yeh et $a l^{(4)}$ reported a case of acromioclavicular dislocation associated with a fracture in the diaphysis of the clavicle that was treated with open reduction of the joint and fixation with two crossed Steinmann wires, without any attention to the coracoclavicular ligaments or the clavicular fracture. The patient presented satisfactory evolution.
Wurtz et $a l^{(5)}$ reported on four cases (three type IV dislocations and one type II): two of the type IV cases were fixed using a coracoclavicular screw, the other type IV cases was treated using transarticular Steinmann wires and the type II case was treated non-surgically. The clavicular fractures were not fixed in any of the four cases and all the patients evolved with good functional results.

In 1995, Heinz et al apud Yeh et al ${ }^{(4)}$ described a similar case that was treated non-surgically, in which the patient was capable of returning to his previous level of physical activity.

In 2002, Juhn and Simonian ${ }^{(6)}$ reported a case of type VI acromioclavicular dislocation that was associated with a greenstick fracture of the diaphysis of the clavicle, which was also treated non-surgically. The patient evolved satisfactorily and returned to his previous level of physical activity, although radiographs produced 10 months after the trauma revealed slight osteolysis of the distal clavicle.

In 2007, Wade et $a l^{(3)}$ described a case of complete acromioclavicular dislocation with posterior and inferior displacement of the clavicle and intact coracoclavicular ligaments (not classifiable in the system of Rockwood et $\left.a l^{(1)}\right)$, associated with a medial epiphyseal fracture of the clavicle with anterosuperior sternoclavicular dislocation, in a 20-year-old patient. The treatment consisted of open reduction and internal fixation of the acromioclavicular joint using one Knowles wire and one Kirschner wire, and then reconstruction of the ligament capsule of the sternoclavicular joint using an anchor. The clinical evolution was unremarkable and the patient recovered completely.

Yeh et $a l^{(4)}$ reported a case of type IV acromioclavicular dislocation associated with a displaced diaphyseal fracture of the clavicle. This case was treated by means of coracoclavicular reconstruction, using an allograft from the semitendinosus tendon, which was passed underneath the coracoid process and sutured around the clavicle, followed by osteosynthesis of the clavicle using a premolded plate. The patient recovered completely and was capable of resuming his previous physical activities.

In all the cases described above, except for the last one, a second procedure was needed in order to remove the synthesis material.

In the case described here, choosing immediate surgical treatment seemed to us to be logical, given that type IV acromioclavicular dislocations have a formal surgical 
indication $^{(1,2)}$. Moreover, there is growing evidence supporting surgical treatment for certain displaced and/ or shortened fractures of the clavicle, especially those affecting younger and/or physically active individuals. Our patient evolved with rapid and progressive alleviation of the pain and improvement of mobility, despite partial loss of the reduction of the acromioclavicular joint, which was observed radiographically two months after the operation. There were no signs of displacement of the anchors that had been fixed in the coracoid process. This partial loss of the reduction probably occurred insidiously, perhaps through tearing of one of the ties and/or through attenuation of the trapezius-deltoid fascia, which may suggest that the coracoclavicular tie and the reconstruction of the trapezius-deltoid fascia alone may be insufficient to bear the enormous tension caused by the weight of the upper limb on the acromioclavicular region. With hindsight, it is possible that placement of one or two temporary transarticular Steinmann wires to increase the rigidity of the fixation while the soft tissues healed might have avoided this complication. The main disadvantages of using transarticular wires are the need to perform a second procedure to remove them, a possibly greater predisposition towards acromioclavicular arthrosis later on and the possibility that the wires might break or migrate into prime structures in the shoulder or in other localities, which could lead to catastrophic complications ${ }^{(6)}$. At the time when we noticed the subluxation, we dismissed the possibility of a second intervention to correct it, on the grounds that the subluxation did not present any clinical or cosmetic repercussions, and supported by the well- known fact that acromioclavicular subluxation mostly has a satisfactory clinical course with non-surgical treatment $^{(1)}$.

After 12 months of postoperative evolution, the patient was totally asymptomatic and did not present any important cosmetic abnormality. He had returned to his previous level of physical activity. He did not have any functional limitation, and the strength and resistance of the scapular belt were preserved. A tomographic scan produced at that time did not show any degenerative changes in the acromioclavicular joint.

We reported an extremely rare case of a displaced extraarticular fracture of the medial extremity of the clavicle that was associated with type IV acromioclavicular dislocation, in a male adult. Despite the absence of clear guidelines in the literature for dealing with these combined lesions, we chose to perform surgical stabilization of the two lesions on the basis of the current literature, which recommends surgical treatment for type IV acromioclavicular dislocations in adults ${ }^{(6)}$ and for some highly displaced and/or shortened fractures of the clavicle. This last recommendation is made especially in cases of young and/or physically active individuals.

The techniques used for fixation of the two lesions find backing in the literature ${ }^{(8,10)}$, but it is possible that addition of one or two temporary transarticular Kirschner or Steinmann wires might have provided greater rigidity of reconstruction during the healing of the soft tissues, and might have avoided the subluxation of the acromioclavicular joint that was observed two months after the operation. However, this event did not interfere with the good functional and cosmetic result.

\section{REFERENCES}

1. Rockwood CA, Williams G, Young D. Disorders of the Acromioclavicular Joint. In: Rockwood CA, Matsen FA 3rd. editors. The shoulder. 2nd ed. Philadelphia: Saunders; 1998. p. 483-553.

2. Trainer G, Arciero RA, Mazzocca AD. Practical management of grade III acromioclavicular separations. Clin J Sport Med. 2008;18(2):162-6.

3. Wade AM, Barrett MO, Crist BD, Della Rocca GJ, Kane SM. Medial clavicular epiphyseal fracture with ipsilateral acromioclavicular dislocation a case report of panclavicular fracture dislocation. J Orthop Trauma. 2007;21(6):418-21.

4. Yeh PC, Miller SR, Cunningham JG, Sethi PM. Midshaft clavicle fracture and acromioclavicular dislocation: a case report of a rare injury. J Shoulder Elbow Surg. 2009;18(5):e1-4.

5. Wurtz LD, Lyons FA, Rockwood CA Jr. Fracture of the middle third of the clavicle

and dislocation of the acromioclavicular joint. A report of four cases. J Bone Joint Surg Am. 1992;74(1):133-7.

6. Juhn MS, Simonian PT. Type VI acromioclavicular separation with middle-third clavicle fracture in an ice hockey player. Clin J Sport Med. 2002;12(5):315-7.

7. Nuber GW, Bowen MK. Acromioclavicular Joint Injuries and Distal Clavicle Fractures. J Am Acad Orthop Surg. 1997;5(1):11-8.

8. Mazzocca AD, Arciero RA, Bicos J. Evaluation and treatment of acromioclavicular joint injuries. Am J Sports Med. 2007;35(2):316-29.

9. Breslow MJ, Jazrawi LM, Bernstein AD, Kummer FJ, Rokito AS. Treatment of acromioclavicular joint separation: suture or suture anchors? J Shoulder Elbow Surg. 2002;11(3):225-9.

10. Hoffler CE, Karas SG. Transacromial erosion of a locked subacromial hook plate: case report and review of literature. J Shoulder Elbow Surg. 2010;19(3):e12-5. 